\title{
Blinking Extraction in Eye gaze System for Stereoscopy Movies
}

https://doi.org/10.1515/phys-2019-0053

Received Jun 21, 2019; accepted Jul 15, 2019

\begin{abstract}
The aim of this paper is to present methods for human eye blink recognition. The main function of blinking is to spread tears across the eye and remove irratants from the surface of the cornea and conjuctiva. Blinking can be associated with internal memory processing, fatigue or activation in central nervous system. There are currently many methods for automatic blink detection. The most reliable methods include EOG or EEG signals. These methods, however, are associated with a decrease in the comfort of the examined person. This paper presents a method to detect blinks with the eye-tracker device. There are currently many blink detection methods for this devices. Two popular eye-trackers were tested in this paper. In addition a method for improving detection efficiency was proposed.
\end{abstract}

Keywords: data science algorithms, stereoscopy, blinking extraction, Eye-gaze systems

PACS: 07.05.Bx, 42.66.Si, 42.66.Ew, 87.19.L-

\section{Introduction}

Gaze motion research has started in 1879 when French ophthalmologist Louis Emile Javal came to a conclusion that observer does not sweep smoothly along the text with his eyes, but with a series of stops and quick saccades [1]. Since his first observation, the eye-tracking devices were developed. Firstly, those devices were very simple, readers had to wear a special type of contact lens with a small opening for the pupil. The lens was attached to a pointer

\footnotetext{
^Corresponding Author: Piotr Napieralski: Institute of Information Technology, Lodz University of Technology, Poland; Email: piotr.napieralski@p.lodz.pl

Anna Rogalska: Institute of Information Technology, Lodz University of Technology, Poland

Filip Rynkiewicz: Institute of Information Technology, Lodz University of Technology, Poland; Email: 173186@edu.p.lodz.pl

Marcin Daszuta, Krzysztof Guzek: Institute of Information Technology, Lodz University of Technology, Poland
}

๑ Open Access. (๐ 2019 A. Rogalska et al., published by De Gruyter. (cc) BY 4.0 License which changed its position following the movements of the eye. The significance of those studies has led to a growth of new, more complicated devices that can automatically measure gaze point. With the rapid advances in information technology, more modern eye-tracking devices were developed. These devices give the possibility of detecting a very complex and dynamic factors in the movie environment. These characteristics allow us to evaluate the experience of the viewer [2]. Using an eye-tracking method to evaluate a viewer's image allows to analyse the experience of the viewer, as well as explore the visual system along with potential processes. The factors that affect the perception of the image are composed of very complex information. Significant is the provocation of the researched group and its influence on the interpretation of the content conveyed in the film image. Measurement of visual discomfort can be done by monitoring the physiological response of the observer. Such reactions include eye pressure, blink frequency, or electromagnetic activity of the brain $[3,4]$.

\section{Human eye anatomy}

Eye is an organ of the visual system, it provides the ability of visual process thus vision. It's origin can be found in photoreceptor proteins that sense light, to simply distinguish between dark and light, found in unicellular organisms. Evolution and adaptation of those simple protein has lead to creation of multiple and very different eye types. Construction of Human eye is not the most complicated in nature, but nevertheless its very sophisticated. For needs of this article the human eye model must by simplified, the diagram can be seen at Figure 1. Most important parts of reduced human eye model consist of:

- Retina is a light-sensitive layer of tissue.

- Pupil is a hole located in the centre of the iris of the eye that allows light to strike the retina.

- Iris is a thin, circular structure in the eye, responsible for controlling the diameter and size of the pupil and thus the amount of light reaching the retina.

- Eye lid is a thin fold of skin that covers and protects the human eye 


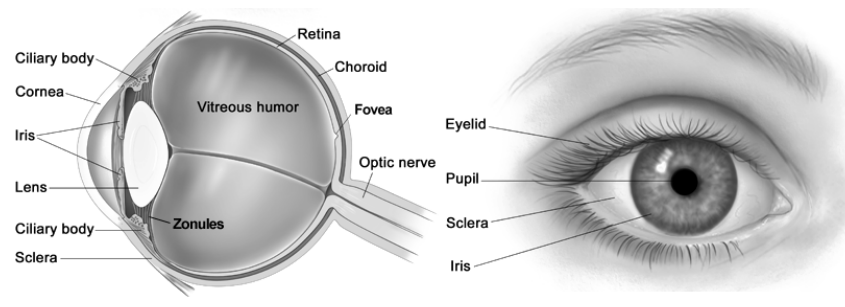

Figure 1: Human eye anatomy.

Source http://www.relativelyinteresting.com/wp-content/uploads/ 2011/06/eye+anatomy.png

\section{Blinking as a factor of visual comfort}

"The eyes are the mirror of the soul and reflect everything that seems to be hidden" [5]. The eyes play a important role in interpreting a person's intentions. Eyes serve as a important channel of information for people. In some cultures, direct eye contact is avoided for fear of confrontation. Through thousands of years of evolution, humanity has learned how to interpret the state of the eyes. Eye movement tracking is important when is interpreting a person's attention. Blinking can be a signal to detect emotional reactions. Bentivoglio et al [6] identified three normal behavioral Blinking Rate patterns and showed that Blinking Rate is more influenced by cognitive processes than by age, eye color, or local factors. Increasing the frequency of blinking very often means nervousness, lying or that people under stress. Eyes are closed for longer when we are troubled, frustrated, or struggling with something emotionally. Sharon Leal et al. [8] tested the blinking rate during and after lying. The experiment consisted of recording blinking a total of 13 liars and 13 truth tellers lied or told the truth. This experiment demonstrated that in situations when lying requires cognitive demand, lying is associated with a decrease in eye blinks followed by a compensatory effect. Blink is also associated with some defense reactions, causing blinking emotions. Blink rate is one of several psychological measures to assess fatigue and mood condition associated with time-on-task. The rate of blinking is increased when the visual task is difficult or more prolonged $[9,10]$. The nature of the blink (blink closure duration or blinking rate) may be affected by cognitive effects. Evaluations of the blinking nature give various parameters of mental fatigue [11]. Blinking can be easily observed when an individual is fatigued. Blinking was the best indicator of fatigue compared with other eye-tracking measures [12]. Tracking of eye gaze and movement is based on searching the pupil centre, pupil ellipse, the shape of eye etc.
Blinking is most often an involuntary act of shutting and opening the eyelid. When it occurs the eye is automatically closed, so the the position of it will be lost. That's why filtering noise caused by blinking is an important task. The ability to measure the frequency of blinking, allows you to check the observer tiredness. In publications [13, 14] has been shown that the blink frequency is significantly increased when observing 3D images. Eye fatigue additionally increased blinking frequency.

In conclusion, it can be assumed that the blinking of the eyes varies depending on the conditions: reading, resting or observing 2D or 3D images. The relationship between blinking and visual discomfort of 3D image observation has been investigated, it is resulting from faulty disparity, speed, and type of motion of the scene objects (circular motion on the image plane, static scenes, and inward motion) [15].

\section{Blinking extraction}

One way of detecting the blink is to use the Electroencephalography (EEG). EEG is an electrophysiological noninvasive monitoring method to record electrical activity of the brain. With the electrodes placed along the surface of the head one can measure voltage fluctuations (brain waves) resulting from ionic current within the large number of neurons. EEG is commonly used for medical diagnostic in order to acquire information about brain electrical activity and mental states. Thanks to the use of currently available portable devices this technique also can be applied for creating Brain Computer Interface (BCI) allowing remote interaction with applications. An example of the applicability of such systems for selected game interaction tasks was presented by [16]. A novel BCI circuit using the emotion fractal analysis method (EFAM) which quantifies emotions based on data obtained from an electroencephalogram (EEG) was develop by Kobayashi and Nakagawa to adjust the speed of an electric wheelchair in proportion to the intensity of the emotion [17]. Detection of eye artifacts such as blinking from EEG data is not a trivial process and therefore there are many efforts to develop an optimal method $[18,19]$. An approach based on the Discrete Wavelet Transform and Neural Network was presented by [20]. Another way of extracting the blink is to use the Electrooculogram (EOG) [21]. EOG is a technique that allows the standing potential between the cornea and the posterior pole of the eye to be recorder by placing pairs of electrodes either above and below the eye or to the left and right of the eye, presented at Figure 2. 


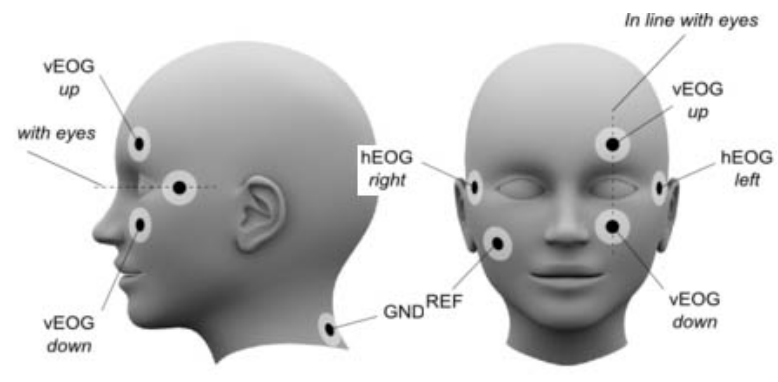

Figure 2: EOG electrodes placement. Source Abdal Kader Shuker EOG Arduino shield

Example EOG signal is presented at Figure 3. This technique is not new but still there are dozens of research where this technique is used [22]. Because of the short distance between sources of the EOG and EEG signals there is a large interference and the amplitude of the EOG signals is only few times greater than EEG signals [23]. Thus, it is also possible to extract eye state data from the EEG signal.

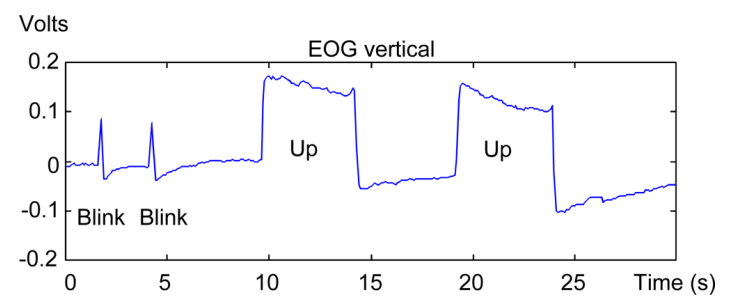

Figure 3: Example of EOG signal.

Source http://www.mdpi.com/sensors/sensors-11-00310/article_ deploy/html/images/sensors-11-00310f6-1024.png

For the purpose of our research Infiniti Flexcomp was used to enable acquisitions of a broad spectrum of brain waves. Each brain wave has different frequency, amplitude and meaning [24]. Signal from the selected frequency range was digitized and processed to emphasize the relevant information. When the eye is moving upward the potential is peeking rapidly, remain horizontal for few seconds and then dip to the previous position. Moving down will provide the opposite signal, there will be fast drop then the signal will remain in the same position for few second before peaking to the middle position again. The blink is the combination of fast up and down movement. So there will be very fast movement, approximately from 100 to 400 milliseconds ("Harvard Database of Useful Biological Numbers"). This can be detected on the EEG signals with some additional filtering operations. The biggest disadvantage of this approach in extracting the blink is that human emotions can affect EEG. In case where blink might be used to detect visual comfort those little unpleasantness could be significant in research, and give noisier data.

\section{Eye tracker}

Another approach to extracting blink is to use eye tracker device. Those apparatus works on base with infra-red or regular cameras, which track the position of eyes, thus they calculate the gaze of human eye.

The most common devices are from Tobii and EyeTribe company. The Tobii X2 is a standalone eye-tracker that can be used in various setups by attaching it to the monitors, laptops or for performing eye tracking on physical objects. It has sampling rate $60 \mathrm{~Hz}$ and system latency under $35 \mathrm{~ms}$ and it is aimed at determining precisely where the participants are looking, the gaze point, timing, duration of fixations and eye movements such as saccades, for example. This device is using Tobii EyeCore algorithm.

Second device is also capable of sampling data in $30 \mathrm{~Hz}$ and $60 \mathrm{~Hz}$ with less than $20 \mathrm{~ms}$ latency at $60 \mathrm{~Hz}$. This equipment has a way worse precision and has more trouble compensating for users that move their head when being tracked.

The output of eye tracker can be seen as the heat map, or as gaze plots. For this research purpose there was no need to use gaze plots, only the heat map was used Figure 4. Those maps show how looking is distributed over the stimulus. There is no information about order of looking in a static heat map (Figure 4).

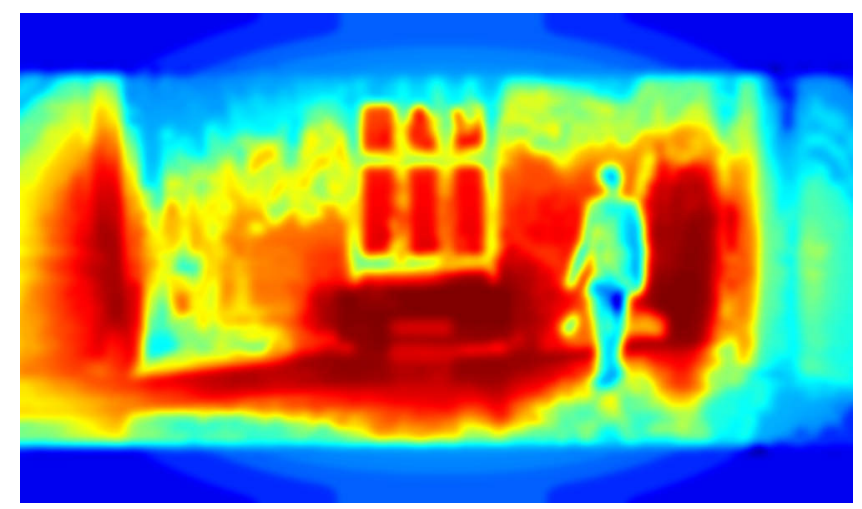

Figure 4: Example of heat map (results of the author's method)

Most trivial approach [25] to blink detection used in eye trackers is to first locate the region of eyes (Figure 5), afterwards to find pupil on this region, by tresholding it on some value (Figure 6). 
Most of the detection algorithms are based on the region of the image. Algorithm verify and classify regions with linear Support-vector machine classifier to extract the candidate region in a given area [26]. Support-vector machine detector scans through this regions using sliding windows (Figure 5).

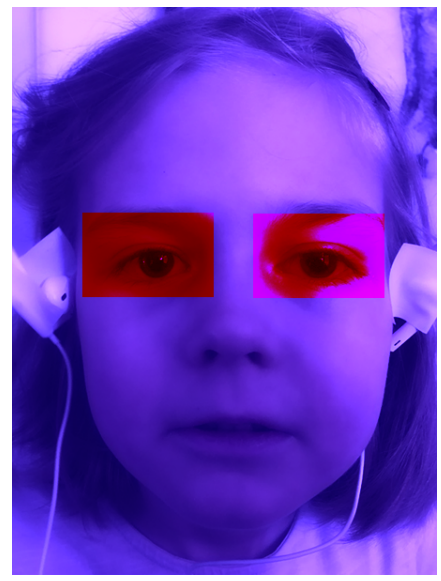

Figure 5: Region proposal by the SVM - Real model which classifies the eyes from the video

Detection of the pupil in the region of interest is made by thresholding. Thresholding is well known method for classify the pixel values in an image. Thresholding is done on gray-scale image, this image has pixel values ranging from $0-255$. Pixels from this region are classify into groups setting a upper and lower bound to each group.

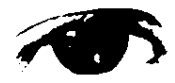

Figure 6: Locate the pupil on image, by threshold image

Output of this operation would be the most dark area on image, assuming that there is only eye on the image. In next frames the operation is repeated. If the area of detected pupil is lower in some value, in example $80 \%$ than in few previous frames, the blink is detected (Figure 7). In [27] the method of calculating blink occurrences has been shown. Based on Facial Landmarks characteristic points $p_{n}$ on eye lids are calculated. On each eye lid the two points are estimated, and based on distance between those points on upper $\left(p_{2} \& p_{3}\right)$ and lower eye $\operatorname{lid}\left(p_{6} \& p_{5}\right)$ the blink is detected. If distance is lower than some threshold the blink is detected.
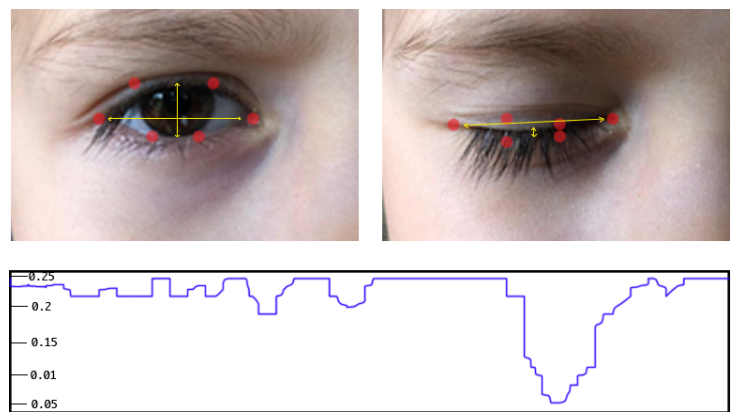

Figure 7: Calculated Facial Landmarks of eye (red points - top), $p$. Blink recognition for with Soukupova et al. method [27]. Bottom Plotting the eye aspect ratio over time.

Another approach is presented at [28]. The detection algorithm is divided into four stages. First the face is recognized using Haar-like features, then the eye region is extracted based on certain human eye geometry dependencies. Blink recognition is based on template matching of opened eye image, acquired at initialization of the system, and current frame of movie. The correlation coefficient between the tempted open eye image and current eye image is calculated using normalized cross correlation method (Figure 8). This coefficient can be treated as openess of the eye, and if the value is lower than predefined threshold value $T L$ for any two consecutive frames, the on-set of the eye-blink is detected. The off-set of the eye-blink is found if the value of the correlation coefficient is greater than the threshold value $T H$.
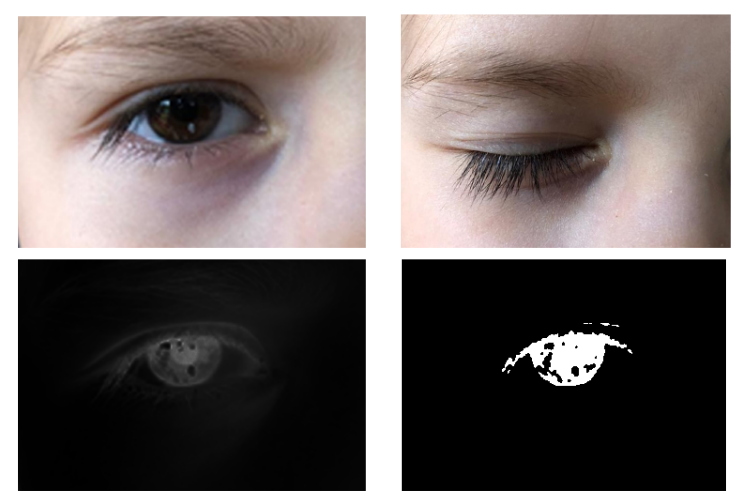

Figure 8: Eye-blink detection by means of difference images. top left: opened eye, top right: closed eye, bottom left: difference image, bottom right: thresholded difference image

Hershman et al.[29] propose a novel blink detection algorithm based on the fluctuations that characterize pupil data. They result from measurement error produced by the eyetracker device. This method finds the onset and offset 
of the blinks on the basis of this noise pattern and its distinctiveness from the eyelid signal. In this moment presented algorithm is more accurate than the others.

There are several approaches to detecting blinks, most of these approaches are not effective and they are burdened with a large number of errors. Popular eye-tracking devices using these methods still have problems with correct blink identification.

\section{Experiment}

In our research we try to identify reliable blinks and eliminating the noise resulting from measuring blanks. During the research both eye-tracker devices, previously described, were tested separately. The main problem with the Eye Tribe, is that it's no longer in production. However, it is still a popular device because of its low price. The system has worked in real-time with constant lighting conditions with similar position of the subject with similar orientations of the head. To increase the reliability of the measurement of the focus points of the viewer's eyes and the correct recording of eye blinks, an additional human face image recorder (GoPro camera) was used to record the face image during the tests. Participated in this research and their task was to watch 5 films each. Every film was from 1:02 to 1:10 minute long, with 10 seconds break between films. In every projection both devices was capturing the eyes, and the face of the examined. Then from data collected by the Eye-Tracker, blink was extracted and manually compared with the film from camera.

For the tested devices under the lighting conditions that prevailed at the test site (daylight falling through the window) was unable to register all blinks.

- the device often interpreted the squinting as a blink,

- the respondents, despite informing them not to move during the study, unfortunately moved on,

- other measurement errors ...

A comparison of the manual analysis of the video recording results and those returned by the EyeTribe indicated that result from the eye-tracker was not as reliable as it was previously thought. The device unexpectedly often interpreted eye loss as a batting factor (Figure 9).

The test results became more reliable after replacing the EyeTribe device with Tobii's X2. Blink reliability tests proved to be much better than the EyeTribe, but still not enough (Figure 10).

The first tests showed a significant advantage of the Tobii's X2 device over EyeTribe. The first measure-

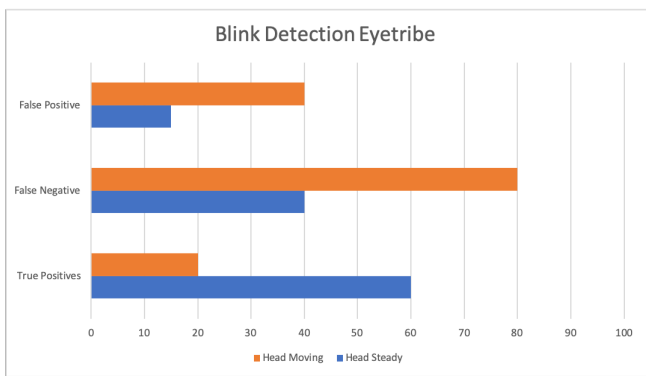

Figure 9: Comparison Charts - Blink Detection for EyeTribe device

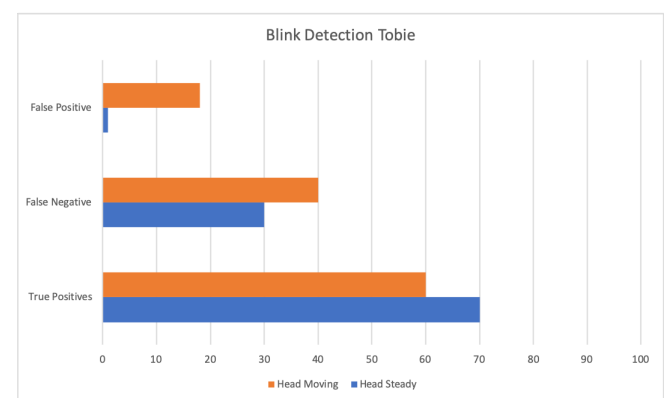

Figure 10: Comparison Charts - Blink Detection for Tobie device

ment showed a large discrepancy between the real blinks (recorded by the camera) and the blinks detected by the eye-tracker system. The effectiveness of blinking detection in the proposed system was $70 \%$. The green dots represent blink onset and offset as detected by our algorithm (Figure 11).

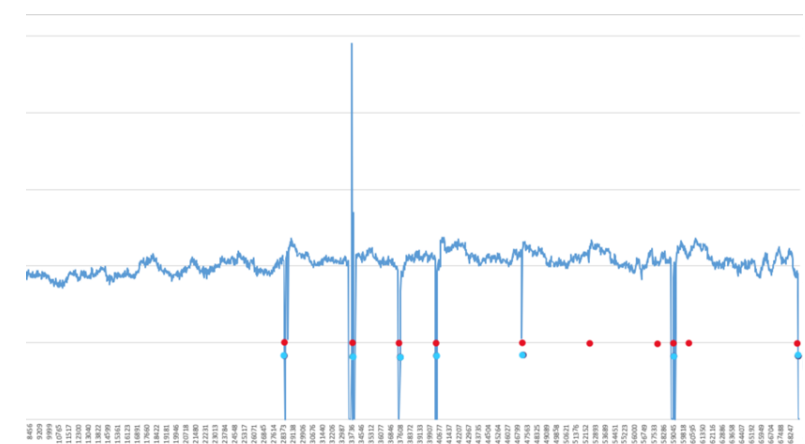

- detected blink pupil diameter - real blink

Figure 11: Eye-blink detection by Tobii's X2 device (with standard SDK). Blue chart is a pupil diameter over the time, blue dots are blinks detected by eye-tracker, red dots are blinks recorded by camera (real blinks)

Our system is used to classify each eye separately (Stereoscopy). Positive examples was collected. Negative examples have been marked as noise samples. This data 
set was used to trained Convolutional Neural Networks ${ }^{1}$. We train a binary classifier between open and close eyes and we use stored noise samples. We used keras library with tensorflow backend. We used filtering for eye blink detection algorithms to increase the effectiveness of blink detection. We noticed better results, increase to almost $90 \%$ (Figure 12). The presented method allows to detect blinks in real time with increased effectiveness.

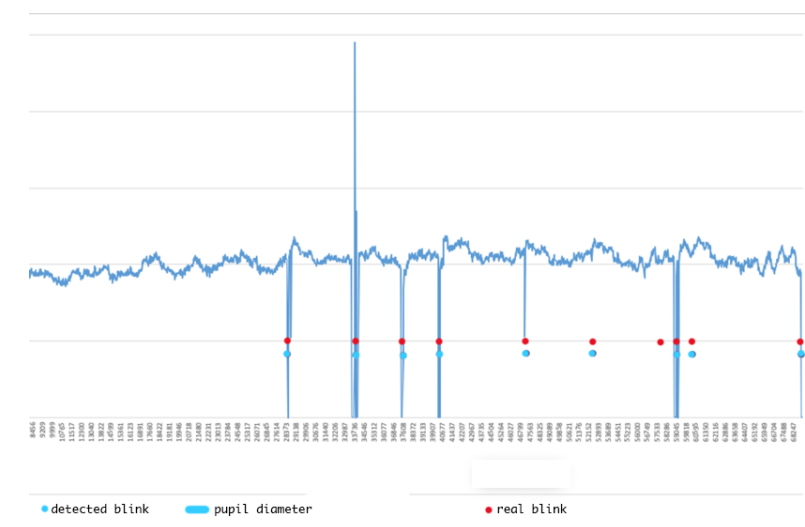

Figure 12: Eye-blink detection by Tobii's X2 device (with CNN). Blue chart is a pupil diameter over the time, blue dots are blinks detected by eye-tracker, red dots are blinks recorded by camera (real blinks)

The designed system was used for preliminary tests. The obtained results were characterized by high noise. The noise resulted from a badly designed test questionnaire. Nevertheless, the results obtained confirmed the theses from other scientific research. It is observed that blinkrate of a person is about 10 to 15 times per minute [6]. The blinkrate is higher when a person is talking, when a person is stressed he tends to blink more. Children do not blink at the same rate like adults. Between each blink is an interval of 2-10 seconds. The performed experiments allow for a full analysis of the blinking frequency. The developed solution was tested on both adults and children. The proposed method is still very sensitive to changing the lighting conditions and to change the position of the head [30]. The introduction of solutions for the correct identification of the pose of the head and the leveling of errors resulting from changes in lighting should significantly improve the efficiency of presented method. Detection of blink frequency is a difficult task for eye-tracker devices. The obtained results allow to assume that the chosen direction is correct.

$1 \mathrm{http}: / / \operatorname{cs} 231 \mathrm{n}$. stanford.edu/
The use of additional sampling methods to detect blinks should increase the effectiveness and reliability of results.

\section{Conclusion}

We propose an efficient method for eye-blinking detection. The method increased the effectiveness of blink detection for popular eye-trackers. The proposed solution increased efficiency to $90 \%$. In our test we found that there is no perfect and reliable solution to extract the blink. The proposed method allows to extend the experimental stand by the ability to blink detection. The correct operation of the large algorithm depends on the lighting conditions, behavior of the examined person and many other factors. Elimination of harmful factors should increase the effectiveness of blink detection. In the presented paper, characteristic errors were detected and eliminated. The algorithm was divided into two phases. The first phase concerned the analysis of the device's operating characteristics. The second phase was based on eliminating common errors detected in the first phase. The algorithm is still very sensitive to changes in the position of the head. Various changes in light intensity also significantly reduce its efficiency.

The designed system in the future is to be used to study the quality of stereoscopic movies. In the further research the connection between blink and emotion will be evaluated. The examination of the viewer's emotions in the cinema is the main goal of the presented system. Of course the EOG perfectly reveals the blink, but the diodes and cables can be disturbing to surveyed, thus the emotion recognition could be noised. On the other hand the eye-tracer device does not always detect the blink, but for user it's less intrusive. In next researches there is a need to create solution for extracting the blink where the method will be indifferent for the user and could identify blink with the best accuracy possible.

\section{References}

[1] Huey E. B., Dearborn W. F., “The psychology of reading an experimental study of the reading pauses and movements of the eye," The American Journal of Psychology, 1907, vol. 18, no. 1, pp. 144-144

[2] Mital P. K., Smith T. J., Hill R. L., Henderson J. M., Clustering of gaze during dynamic scene viewing is predicted by motion, Cognitive Computation, 2010 vol. 3, no. 1, pp. 5-24

[3] Fornalczyk K., P. Napieralski, Szajerman D, Wojciechowski A, Stereoscopic image visual perception, International Journal of Microelectronics and Computer Science, 2015. 
[4] Fornalczyk K., Napieralski P., Szajerman D., Wojciechowski A., Sztoch P., Wawrzyniak J., Stereoscopic image perception quality factors, June 2015 pp. 129-133.

[5] Coelho P., "Manuscript found in accra quotes," 2013.

[6] Bentivoglio A. R., Bressman S. B., Cassetta E., Carretta D.,

[7] Tonali P., Albanese A., Analysis of blink rate patterns in normal subjects, Movement Disorders, 1997, vol. 12, no. 6, pp. 10281034,

[8] Leal S., Vrij A., Blinking during and after lying, Journal of Nonverbal Behavior, 2008 vol. 32, pp. 187-194,

[9] Luckiesh M., Reading and the rate of blinking., Journal of Experimental Psychology, 1947, vol. 37, no. 3, pp. 266-268

[10] Stern J. A., Boyer D., Schroeder D. J., Blink rate as a measure of fatigue: A review., PsycEXTRA Dataset, 1994

[11] Stasi L. L. D., Catena A., Canãs J. J., Macknik S. L., Martinez-Conde S., Saccadic velocity as an arousal index in naturalistic tasks, Neuroscience \& Biobehavioral Reviews, 2013, vol. 37, no. 5, pp. 968-975,

[12] Yamada Y., Kobayashi M., Detecting mental fatigue from eyetracking data gathered while watching video, Artificial Intelligence in Medicine, 2017, pp. 295-304,

[13] Lee E., Heo H., Park K., The comparative measurements of eyestrain caused by $2 \mathrm{~d}$ and $3 \mathrm{~d}$ displays, IEEE Transactions on Consumer Electronics, 2010, vol. 56, no. 3, pp. 1677-1683

[14] Yu J.-H., Lee B.-H., Kim D.-H., Eog based eye movement measure of visual fatigue caused by $2 \mathrm{~d}$ and $3 \mathrm{~d}$ dis- plays, Proceedings of 2012 IEEE-EMBS International Conference on Biomedical and Health Informatics, 2012.

[15] Li J., Barkowsky M., Le Callet P., Visual discomfort is not always proportional to eye blinking rate: Exploring some effects of planar and in-depth motion on 3dtv qoe, 2013.

[16] Szajerman D., Warycha M., Antonik A., Wojciechowski A., Popular brain computer interfaces for game mechanics control, Advances in Intelligent Systems and Computing, 2016, pp. 123-134.

[17] Kobayashi N., Nakagawa M., Bci-based control of electric wheelchair using fractal characteristics of EEG, IEEJ Transactions on Electrical and Electronic Engineering, 2018 vol. 13, no. 12, pp. 1795-1803

[18] Rihana S., Damien P., Moujaess T., EEG-eye blink detection system for brain computer interface, Converging Clinical and Engineering Research on Neurorehabilitation, 2013, pp. 603-608
[19] Opatka, S, Stasiak B., Szajerman D., Wojciechowski A., Multichannel convolutional neural networks architecture feeding for effective EEG mental tasks classification, Sensors, 2018, vol. 18, no. 10 , pp. 3451-3451

[20] Tibdewal M. N., Fate R. R., Ray A., Detection and classification of eye blink artifact in electroencephalogram through discrete wavelet transform and neural network, 2015 International Conference on Pervasive Computing (ICPC), 2015.

[21] Denney D., Denney C., The eye blink electrooculogram., British Journal of Ophthalmology, 1984 , vol. 68, no. 4, pp. 225-228

[22] Yagi T., Kuno,K., Koga K., Mukai T., "Drifting and blinking compensation in electro-oculography (eog) eye-gaze interface," 2006 IEEE International Conference on Systems, Man and Cybernetics, 2006. 16 cites:.

[23] Babus`iak B., Mohylovaj., Eye-blink artifact detection in the EEG,” IFMBE Proceedings, 2009, pp. 1166-1169

[24] Koudelkovaź. , Strmiska M., Introduction to the identification of brain waves based on their frequency, MATEC Web of Conferences, 2018, vol. 210, pp. 5012-5012

[25] Naveed S., Sikander B., Khiyal M., Eye tracking system with blink detection, Journal of Computing, 2012, vol. 4, pp. 50-60

[26] Young-Joo Han J.-S. P., Kim Wooseong, Efficient eye-blinking detection on smartphones: A hybrid approach based on deep learning, Mobile Information Systems, 2018.

[27] Soukupovaí., Cech J., Real-time eye blink detection using facial landmarks, 2016.

[28] Krolak A., Strumiłto P., Eye-blink detection system for humancomputer interaction, Universal Access in the Information Society, 2012, vol. 11, pp. 409-419

[29] Hershman R., Henik A., Cohen N., A novel blink detection method based on pupillometry noise, Behavior Research Methods, 2018, vol. 50, pp. 107-114

[30] Fornalczyk K., Wojciechowski A., Robust face model based approach to head pose estimation, 2017 Federated Conference on Computer Science and Information Systems (FedCSIS), 2017, pp. 1291-1295 